\title{
Neutrophil-to-lymphocyte ratio (NLR) at boundaries of Progressive Supranuclear Palsy Syndrome (PSPS) and Corticobasal Syndrome (CBS)
}

\author{
Piotr Alster, Natalia Madetko, Andrzej Friedman \\ Department of Neurology, Medical University of Warsaw, Warsaw, Poland
}

\begin{abstract}
:
Aim of the study. To examine possible features of neuroinflammation in progressive supranuclear palsy - Richardson syndrome and corticobasal syndrome (CBS).

Clinical rationale for the study. Neutrophil-to-lymphocyte ratio (NLR) is a parameter reflecting inflammation used in numerous branches of medicine. The search for pathogenesis of the diseases partly related to inflammatory processes confirms the need to obtain possible factors which could be relatively easily verified. NLR is a benchmark routinely evaluated in most hospitalised patients.
\end{abstract}

Materials and methods. 23 patients with a clinical diagnosis of PSP-RS, 18 patients with CBS, and 32 healthy controls, were included in the study. Blood samples were assessed in the context of neutrophil and lymphocyte rates. Subsequently, the results were transformed into neutrophil-to-lymphocyte ratio (NLR). The NLRs from each group were statistically assessed using a Kruskal-Wallis test and post-hoc analysis.

Results. Statistical analysis confirmed significant differences in NLR between PSP-RS and control group. No other significant differences were observed.

Clinical implications. The possible use of NLR in the additional examination of atypical parkinsonisms.

Conclusions. To the best of our knowledge, this is the first study comparing this aspect of neuroinflammation in PSP and CBS. It presents NLR as a promising non-specific parameter in neurodegenerative diseases.

Key words: progressive supranuclear palsy, PSP, corticobasal degeneration, CBS, NLR

(Neurol Neurochir Pol 2021; 55 (1): 97-101)

\section{Introduction}

Neutrophil-to-lymphocyte ratio (NLR) is a non-specific parameter which has been assessed for almost two decades. It was initially introduced in the evaluation of intensive care unit patients. With the progress of knowledge, NLR has become one of the indicators of increased mortality [1]. Recent studies have evaluated the role of NLR in the examination of COVID-19 [2]. It has also been analysed in the context of ageing and various chronic and acute diseases. The role of NLR in the examination of movement disorders has been evaluated in Parkinson's Disease (PD), Progressive Supranuclear Palsy
(PSP) and Essential Tremor (ET). The results show that PSP is associated with higher NLR than PD. No differences in the NLR among PD subtypes were detected [3]. On the other hand, NLR was shown as significantly increased in PSP compared to PD [4]. ET was not associated with any significant changes in the level of NLR [5]. None of the studies considered blood parameters in Corticobasal Syndrome (CBS). Moreover, the issue of these quotients is not mentioned in the contemporary diagnostic criteria [6].

Progressive supranuclear palsy is a neuropathological term describing aggregation of microtubule-associated protein tau 4-repeats isoform (MAP/tau - 4R) with depositions in neurons 
and glial cells. Microscopically, neurofibrillary tangles associated with tufted astrocytes, oligodendroglial coiled bodies and threads, all comprising 4-R tau, are described as typical for PSP pathology. Lesions are located mainly in the rhombencephalon [7]. Corticobasal degeneration (CBD) is a neuropathological entity describing 4-R tau neuronal inclusions, threads, coiled bodies and, and this is the most characteristic, astrocytic plaques located predominantly in the forebrain [7]. Ante mortem diagnosing enables the identification of only clinical syndromes like progressive supranuclear palsy syndrome (PSPS) and CBS which may proceed not only from PSP and CBD pathologies accordingly.

The boundaries of CBS and PSPS are evaluated on various grounds. Due to overlapping clinical manifestations, interpreting PSPS and CBS as independent entities has become less obvious [8-10]. Contemporary criteria of PSP diagnosis mention PSP-CBS - a syndrome with mixed symptomatology, however multiple cases show dominant PSP or CBS syndrome, with insufficient evidence to diagnose PSP-CBS [11]. Contemporary literature has devised the term '4-repeat tauopathy' (4RT), which is becoming a more popular diagnosis especially in patients with no evident symptomatology. The term is associated with a clinical diagnosis of joint PSP and CBD [8]. The evaluation of the accuracy of this diagnostic term was based on the examination of 195 patients with pathologically confirmed PSP and 55 with CBD. A total of 161 patients with non 4-repeat tauopathies with Multiple System Atrophy, Parkinson's Disease and 4RT-negative Frototemporal Lobe Dementia were additionally examined in the comparison. The authors of this research suggest that the diagnosis of 'possible PSP-CBS' may be doubtful in obtaining any benefit in the examination of $4 \mathrm{RT}$. The term ' $4 \mathrm{RT}$ ' has been further analysed in the context of treatment perspectives and is interpreted as feasible in clinical trials $[8,12]$.

\section{Clinical rationale for study}

Recent research has shown possible differences in neuroimaging. However, evolving concepts regarding the pathogenesis of both entities show the necessity of the analysis of neuroinflammation as a possible common feature of PSP and CBD, which are pathologies associated with PSPS and CBS $[13,14]$. The activation of microglia is shown to be one aspect of neurodegeneration [15]. Though the described entities are syndromes representing various pathologies, the correlation between pathophysiology and symptomatology remains unexplored.

The aim of this study was to assess whether NLR is a non-specific parameter which could differentiate between PSP, CBS and healthy subjects. The results of the study could contribute to the discussion as to whether, in terms of NLR, the boundaries between PSP and CBS are relevant.

\section{Materials and methods}

23 patients (15 male, eight female) with a clinical diagnosis of PSP Richardson (PSP-RS), 18 patients (two male, 16 female) with a clinical diagnosis of CBS, and 32 patients (18 male, 14 female) who formed a control group, were included in the study. The age of patients with PSP-RS ranged from 61 to 82, in CBS from 56 to 86, and in the control group from 64 to 85 . All the groups were age matched. All the included patients with PSP-RS and CBS matched the current criteria of diagnosis $[6,11]$. All the patients diagnosed with PSP-RS demonstrated oculomotor dysfunction, bradykinesia, disturbances related to postural reflexes, and cognitive and speech deficiencies. Patients with CBS demonstrated asymmetric limb rigidity, axial rigidity, postural instability, bradykinesia, clumsy limbs, and asymmetrical manifestation of symptoms. Both groups experienced falls. All the patients were diagnosed with probable or possible PSP-RS or CBS. All the patients were evaluated using Unified Parkinson's Disease Rating Scale - III to verify possible response to levodopa treatment. None of the patients included in the study had experienced any improvement after levodopa treatment. The duration of the disease varied from 2-6 years. No pathological examination was conducted. Patients with active inflammatory processes related to infectious, autoimmunological and neoplasmatic diseases were excluded from the study.

Every patient evaluated in the study underwent complete blood count (CBC) assessment, which was examined during their hospitalisation in the Department of Neurology. The $\mathrm{CBC}$ of the control group was evaluated in the occupational medicine department during regular check-ups. Each CBC enabled the analysis of the number of neutrophils and lymphocytes. The obtained data from each was automatically put into a database and was subsequently anonymously analysed. Further analysis was based on the age of the patient, sex, diagnosis, number of neutrophils, number of lymphocytes, NLR, and mediana of NLR in each group (Tab. 1). All the obtained data regarding NLR was evaluated using Statistica software, using a Kruskal-Wallis test to show possible differences of NLR between the three groups - the PSP-RS, CBS and control. The Kruskal-Wallis test was used due to the non-gaussian distribution in the three examined groups. The differences confirmed in Kruskal-Wallis were subsequently analysed using post-hoc Dunn test. Additionally, in order to obtain groups which were age-matched, statistical analysis was used to avoid the impact of age on possible differences between the groups.

\section{Results}

The evaluation using a Kruskal-Wallis test showed significant differences in the comparison of control groups and patients with PSP-RS ( $\mathrm{p}=0.006413$ ) (Fig. 1, Tab. 2). No other 
Table 1. Descriptive statistics

\begin{tabular}{|c|c|c|c|c|c|c|c|c|c|}
\hline & \multirow[t]{2}{*}{$\begin{array}{l}\text { Number of } \\
\text { individuals }\end{array}$} & \multicolumn{2}{|c|}{$\begin{array}{c}\text { Gender } \\
\text { proportions }\end{array}$} & \multirow[t]{2}{*}{$\begin{array}{c}\text { AGE } \\
\text { Median }\end{array}$} & \multirow[t]{2}{*}{$\begin{array}{c}\text { AGE } \\
\text { Range }\end{array}$} & \multirow[t]{2}{*}{$\begin{array}{c}\text { NLR } \\
\text { Mean }\end{array}$} & \multirow[t]{2}{*}{$\begin{array}{c}\text { NLR } \\
\text { Median }\end{array}$} & \multirow[t]{2}{*}{$\begin{array}{c}\text { NLR } \\
\text { Range }\end{array}$} & \multirow{2}{*}{$\begin{array}{c}\text { NLR } \\
\text { Standard } \\
\text { Deviation }\end{array}$} \\
\hline & & Male & Female & & & & & & \\
\hline PSP-RS & 23 & 15 & 8 & 73 & $61-82$ & 2.88 & 2.69 & $0.79-7.97$ & 1.66 \\
\hline CBS & 18 & 16 & 2 & 71 & $56-86$ & 2.13 & 2.18 & $1.05-2.84$ & 0.53 \\
\hline Control & 32 & 18 & 14 & 71 & $64-85$ & 1.92 & 1.72 & $1.08-4.87$ & 0.84 \\
\hline
\end{tabular}

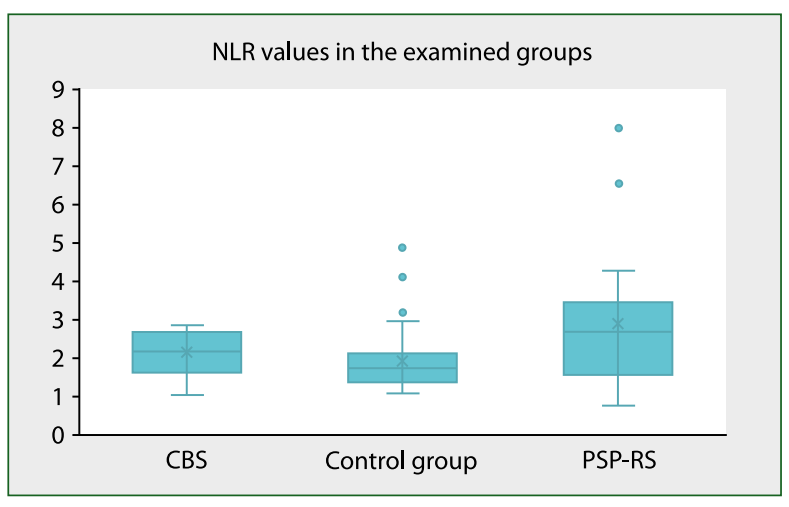

Figure 1. Range of values within each group

Table 2. Comparison of mean NLR values in each group. Post hoc $p$ values (differences in NLR between groups)

\begin{tabular}{lccc|} 
& CBS & $\begin{array}{c}\text { Control } \\
\text { group }\end{array}$ & PSP-RS \\
\hline CBS & & $\begin{array}{c}0.295995 \\
(\mathbf{0 . 4 6 )}\end{array}$ & $\begin{array}{c}0.786741 \\
(\mathbf{0 . 7 5 )}\end{array}$ \\
Control group & 0.295995 & & 0.006413 \\
& $(\mathbf{0 . 4 6 )}$ & & $\mathbf{( 0 . 9 6 )}$ \\
PSP-RS & 0.786741 & 0.006413 & \\
& $\mathbf{( 0 . 7 5 )}$ & $\mathbf{( 0 . 9 6 )}$ & \\
& & & \\
& & &
\end{tabular}

significant differences were observed in the study ( $p>0.05)$. The differences between CBS and control were meaningly beyond the level of significance $-\mathrm{p}=0.295995$. NLR did not show a differentiating potential in the examination of PSP-RS and CBS (Tab. 2).

\section{Discussion}

The obtained results probably suggest that PSP-RS and CBS are factors increasing NLR. Only in PSP-RS did the increase of NLR obtain a significant difference compared to the control group. The increase of NLR in CBS was less pronounced, and was not associated with significant differences compared to the control group. The increase of NLR in both syndromes (PSP-RS and CBS) diminishes the possibility of significant differences between these clinical entities.
Regarding the lack of possibility of pathological verification of the diagnoses, the singular effect in PSP-RS could be related to the fact that PSP-RS is highly predictive for PSP pathology [16]. The CBS correlation with possible CBD pathology is not as common, being associated with about 55\% of CBS cases [17]. The fact that PSP-RS is more likely to be a manifestation of $4 \mathrm{RT}$, whereas there was a lack of significant differences between CBS and the control group, could be partly explained by the diverse pathological basis. Additionally, CBS may be based on 4RT as PSP, CBD, Agyrophilic Grain Disease, Globular Glial Tauopathies, 3-repeat tauopathies as Frontotemporal Dementia and 3/4R tauopathies as Alzheimer's Disease and Primary Age-Related Tauopathy [18].

To the best of our knowledge, this is the first study evaluating patients with CBS in such a context. The results concerning PSP-RS are convergent with those obtained in a study by Inci et al, however in this study PSP was a homogenic group, without the indication of the disease's variants [4].

The potentially underlying mechanisms which are related to the increase of NLR in the presented tauopathies may be related to microglial activation. Its role in the neurodegeneration in atypical parkinsonisms is not fully understood, and in the case of CBS it is practically unexplored. Microglial activation is interpreted as an inflammatory explanation for neurodegeneration [13]. Most studies have concentrated on the association of tauopathic pathologies and neuroinflammation [13]. The correlation between neuroinflammation and clinical manifestations (PSPS or CBS) is unexplored [13].

The results from our study show that atypical parkinsonism based on tauopathic pathology is a feature causing an increase of inflammatory parameters. However, this does not provide information as to whether it is related to the cause of the disease, or perhaps more likely to its effect.

We are aware of the limitations of our study such as the relatively small number of patients. However, various studies evaluating other features, possibly facilitating the examination of CBS, have been based on smaller numbers of patients $[9,10,20]$. The disparity between the number of male and female patients in the CBS group is related to the rarity of this syndrome. There is no information on the impact of sex. Additionally, it should be stressed that our study is based only on clinical manifestations and does not examine the 
pathological background. This factor could possibly impact upon the results of patients with CBS, due to the heterogenic pathological basis of this syndrome. All of the examined patients are alive. Moreover, the main intention of the authors was to evaluate accessible methods in vivo. The NLR is also a non-specific method, providing information on the ongoing inflammation. However, it is examined routinely and can be evaluated rapidly and possibly provide general information.

\section{Clinical implications/future directions}

This study shows that $4 \mathrm{RT}$ may be a factor accelerating inflammatory processes, as well as a consequence of these reactions. The obtained results contribute to the discussion on the pathogenesis of 4RT. Another issue emerging from this study is the controversy as to whether to regard PSPS and CBS as separate entities. The boundaries between the diseases are often questioned due to their overlapping symptomatology, especially PSP-RS and CBS. The recent criteria of diagnosis of PSP show PSP-CBS syndrome. This seemed to be a way of diagnosing ambiguous cases. Further analyses of PSP and CBS symptomatology led to the introduction of the diagnosis of '4-repeat tauopathy' [8]. Earlier studies conducted by our research team revealed similar results in neurological and neuropsychological examination and neuroimaging. Patients diagnosed with advanced PSP-RS presented postural deficits and oculomotor dysfunction accompanied by asymmetrical syndrome with limb dystonia. Asymmetrical atrophy tended to occur with midbrain and cerebellar peduncle decreases of volume. Additionally, no significant differences in perfusion were detected $[9,10,20]$.

The issue of NLR seems to be one of many factors in this discussion. The evaluation of neuroinflammatory factors in PSPS and CBS requires further research based on larger groups of patients. Studies evaluating the role of inflammatory processes in CBS should verify the possible impact of different pathologies standing behind this syndrome.

Ethics: The Bioethical Committee of the Medical University of Warsaw approved this study. The number of the approval is AKBE151/2020.

Conflict of interest: None.

\section{References}

1. Fest J, Ruiter TR, Groot Koerkamp B, et al. The neutrophil-to-lymphocyte ratio is associated with mortality in the general population: The Rotterdam Study. Eur J Epidemiol. 2019; 34(5): 463-470, doi: 10.1007/ s10654-018-0472-y, indexed in Pubmed: 30569368.

2. Li X, Liu C, Mao Z, et al. Predictive values of neutrophil-to-lymphocyte ratio on disease severity and mortality in COVID-19 patients: a systematic review and meta-analysis. Crit Care. 2020; 24(1): 647, doi: 10.1186/s13054-020-03374-8, indexed in Pubmed: 33198786.

3. Ataç Uçar C, Gökçe Çokal B, Ünal Artık HA, et al. Comparison of neutrophil-lymphocyte ratio (NLR) in Parkinson's disease subtypes.
Neurol Sci. 2017; 38(2): 287-293, doi: 10.1007/s10072-016-27588, indexed in Pubmed: 27837368.

4. Inci I, Kusbeci OY, Eskut N. The neutrophil-to-lymphocyte ratio as a marker of peripheral inflammation in progressive supranuclear palsy: a retrospective study. Neurol Sci. 2020; 41(5): 1233-1237, doi: 10.1007/s10072-019-04208-4, indexed in Pubmed: 31901125.

5. Tak AZ, Sengül Y. Evaluation of neutrophil-to-lymphocyte ratio and platelet-to-lymphocyte ratio in essential tremor. Ideggyogy Sz. 2019; 72(1-2): 33-38, doi: 10.18071/isz.72.0033, indexed in Pubmed: 30785244

6. Armstrong MJ, Litvan I, Lang AE, et al. Criteria for the diagnosis of corticobasal degeneration. Neurology. 2013; 80(5): 496-503, doi: 10.1212/WNL.0b013e31827f0fd1, indexed in Pubmed: 23359374.

7. Kovacs GG. Invited review: Neuropathology of tauopathies: principles and practice. Neuropathol Appl Neurobiol. 2015; 41(1): 3-23, doi: 10.1111/nan.12208, indexed in Pubmed: 25495175.

8. Respondek G, Grimm MJ, Piot I, et al. Movement Disorder Society-Endorsed Progressive Supranuclear Palsy Study Group. Validation of the movement disorder society criteria for the diagnosis of 4-repeat tauopathies. Mov Disord. 2020; 35(1): 171-176, doi: 10.1002/ mds.27872, indexed in Pubmed: 31571273.

9. Alster P, Nieciecki M, Koziorowski D, et al. Is brain perfusion a differentiating feature in the comparison of Progressive Supranuclear Palsy Syndrome (PSPS) and Corticobasal Syndrome (CBS)? J Clin Neurosci. 2020; 77: 123-127, doi: 10.1016/j.jocn.2020.05.005, indexed in Pubmed: 32389545.

10. Alster P, Krzyżanowska E, Koziorowski D, et al. Difficulties in the diagnosis of four repeats (4R) tauopathic parkinsonian syndromes. Neurol Neurochir Pol. 2018; 52(4): 459-464, doi: 10.1016/j. pjnns.2018.06.002, indexed in Pubmed: 30025721.

11. Iankova V, Respondek G, Saranza G, et al. Clinical diagnosis of progressive supranuclear palsy: The movement disorder society criteria. Mov Disord. 2017; 32(6): 853-864, doi: 10.1002/mds.26987, indexed in Pubmed: 28467028.

12. VandeVrede L, Ljubenkov PA, Rojas JC, et al. Four-Repeat Tauopathies: Current Management and Future Treatments. Neurotherapeutics. 2020 [Epub ahead of print], doi: 10.1007/s13311-020-00888-5, indexed in Pubmed: 32676851.

13. Alster P, Madetko N, Koziorowski D, et al. Microglial Activation and Inflammation as a Factor in the Pathogenesis of Progressive Supranuclear Palsy (PSP). Front Neurosci. 2020; 14: 893, doi: 10.3389/ fnins.2020.00893, indexed in Pubmed: 32982676.

14. Henkel K, Karitzky J, Schmid M, et al. Imaging of activated microglia with PET and [11C]PK 11195 in corticobasal degeneration. Mov Disord. 2004; 19(7): 817-821, doi: 10.1002/mds.20040, indexed in Pubmed: 15254941.

15. Ishizawa K, Dickson DW. Microglial activation parallels system degeneration in progressive supranuclear palsy and corticobasal degeneration. J Neuropathol Exp Neurol. 2001; 60(6): 647-657, doi: 10.1093/jnen/60.6.647, indexed in Pubmed: 11398841.

16. Josephs KA. Key emerging issues in progressive supranuclear palsy and corticobasal degeneration. J Neurol. 2015; 262(3): 783-788, doi: 10.1007/s00415-015-7682-y, indexed in Pubmed: 25701010.

17. Wadia PM, Lang AE. The many faces of corticobasal degeneration. Parkinsonism Relat Disord. 2007; 13 Suppl 3: S336-S340, doi: 10.1016/S1353-8020(08)70027-0, indexed in Pubmed: 18267261.

18. Irwin DJ. Tauopathies as clinicopathological entities. Parkinsonism Relat Disord. 2016; 22 Suppl 1: S29-S33, doi: 10.1016/j.parkreldis.2015.09.020, indexed in Pubmed: 26382841. 
19. Valotassiou V, Papatriantafyllou J, Sifakis N, et al. Brain perfusion SPECT with Brodmann areas analysis in differentiating frontotemporal dementia subtypes. Curr Alzheimer Res. 2014; 11(10): 941-954, doi: 10.2174/1567205011666141107125104, indexed in Pubmed: 25387340.
20. Alster P, Nieciecki M, Koziorowski DM, et al. Thalamic and cerebellar hypoperfusion in single photon emission computed tomography may differentiate multiple system atrophy and progressive supranuclear palsy. Medicine (Baltimore). 2019; 98(30): e16603, doi: 10.1097/ MD.0000000000016603, indexed in Pubmed: 31348305. 